\title{
Viscous Nonlinear Dynamics of Twist and Writhe
}

\author{
Raymond E. Goldstein, ${ }^{1,2}$ Thomas R. Powers, ${ }^{1}$ and Chris H. Wiggins ${ }^{3}$ \\ ${ }^{1}$ Department of Physics, University of Arizona, Tucson, Arizona 85721 \\ ${ }^{2}$ Program in Applied Mathematics, University of Arizona, Tucson, Arizona 85721 \\ ${ }^{3}$ Department of Physics, Princeton University, Princeton, New Jersey 08544
}

(Received 9 February 1998)

\begin{abstract}
Exploiting the "natural" frame of space curves, we formulate an intrinsic dynamics of a twisted elastic filament in a viscous fluid. Coupled nonlinear equations describing the temporal evolution of the filament's complex curvature and twist density capture the dynamic interplay of twist and writhe. These equations are used to illustrate a remarkable nonlinear phenomenon: geometric untwisting of open filaments, whereby twisting strains relax through a transient writhing instability without axial rotation. Experimentally observed writhing motions of fibers of the bacterium B. subtilis [N. H. Mendelson et al., J. Bacteriol. 177, 7060 (1995)] may be examples of this untwisting process. [S0031-9007(98)06244-9]
\end{abstract}

PACS numbers: 87.10.+e, 02.40.-k, 03.40.Dz, 36.20.Ey

As D’Arcy Thompson emphasized [1], nature abounds with examples of the balance between growth and form. The balance is particularly manifest in the low Reynolds number regime without inertia, where the motion of organisms is generally dominated by dissipation and form is a consequence of elasticity. The coupling between these two has been termed "elastohydrodynamics."

Possibly the most common "form" of subcellular dynamics is the elastic filament: rodlike macromolecules perform a multitude of structural and biological roles. To gain some intuition for dynamics at this scale, the case of the elastic filament is thus a fruitful starting point [2]. As a motivating example, we recall Mendelson's discovery of shape instabilities exhibited by a mutant strain of the bacterium B. subtilis [3]. Filaments of cells form as the rodshaped bacteria fail to separate upon dividing; beyond a critical length, a filament buckles, twisting around itself to form a plectoneme (see Fig. 1). It is thought that this instability arises from nonequilibrium twisting stresses generated in the cell walls through the process of growth $[3,4]$. As the bacteria continue to grow, this process is iterated, leading to a hierarchy of supercoils. The handedness of the coils may be tuned with temperature or ionic concentrations. Since the filament ends are generally free and thermal fluctuations are negligible, this supercoiling is fundamentally different from that of torsionally stressed DNA that is closed or has pinned ends [5]. As a first step toward understanding these highly nonlinear phenomena, we develop here an intrinsic deterministic formulation of twisted elastic filament motion, and apply it supercoiling of $B$. subtilis. Various elements of this formulation have appeared in previous work on closed elastic rods without dissipation [6], where a dynamic (rather than energetic [7]) coupling between twist and bend degrees of freedom has been recognized [8]. In the inertialess case, a geometric argument for this coupling has been recently proposed [9], and its qualitative consequences have been explored [10]. In presenting a general formulation of filament dy- namics, we explicitly derive this twist-bend coupling and illustrate the qualitatively new physics of open filaments in a viscous medium.

An important feature of our treatment is the parametrization of the filament. Although the "Euclidean" representation based on the position vector $\mathbf{r}(s)$ is convenient for linear stability analysis about simple shapes, it makes the full nonlinear problem unnecessarily complicated. As exploited by Kirchhoff [11], the Euler angles of rigid body motion are better suited for elastic equilibria. This parametrization singles out a particular space-fixed reference frame and thus is partly extrinsic. An intrinsic representation expresses the rate of change of a frame (e.g., the material or Frenet-Serret frames) at each point of the rod in terms of the frame itself [12], and is simplified by using the "natural frame" associated to the centerline. First used by Darboux [13] and later by Hasimoto in his study of vortex filament motion [14], this frame has been applied to the dynamics of space curves with bending but no twisting elasticity [15].

The configuration of an elastic rod may be described by a material orthonormal frame $\left\{\hat{\mathbf{e}}_{1}, \hat{\mathbf{e}}_{2}, \hat{\mathbf{e}}_{3}\right\}$ [12]: we choose $\hat{\mathbf{e}}_{3}$ to point along the tangent, $\hat{\mathbf{e}}_{1}$ to point from the centerline to a painted stripe on the rod surface that

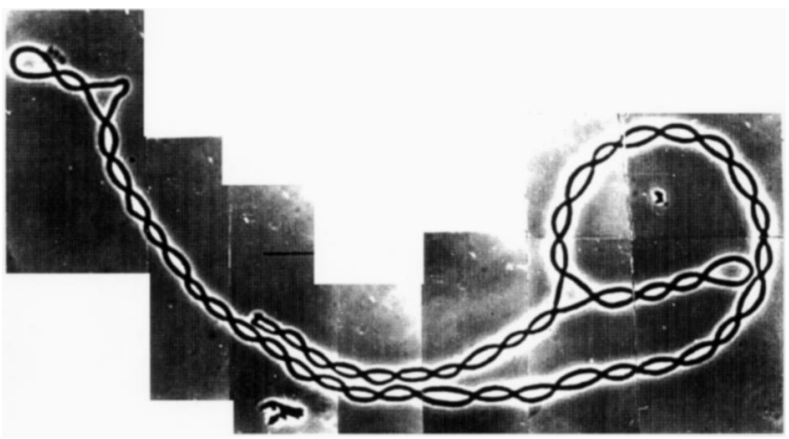

FIG. 1. Supercoiled filament of B. subtilis [3]. Scale bar is $10 \mu \mathrm{m}$. Image courtesy of N. H. Mendelson. 
connects one end to the other, and $\hat{\mathbf{e}}_{2}=\hat{\mathbf{e}}_{3} \times \hat{\mathbf{e}}_{1}$. The strains $\boldsymbol{\Omega}$ are given by the rate of rotation of this frame with arclength $s, \partial_{s} \hat{\mathbf{e}}_{i}=\boldsymbol{\Omega} \times \hat{\mathbf{e}}_{i}$. To quadratic order in $\boldsymbol{\Omega}$, the elastic energy of a homogeneous isotropic rod is

$$
\mathcal{E}=\frac{A}{2} \int d s\left(\Omega_{1}^{2}+\Omega_{2}^{2}\right)+\frac{C}{2} \int d s \Omega_{3}^{2}-\int d s \Lambda,
$$

where $\Omega_{i}=\boldsymbol{\Omega} \cdot \hat{\mathbf{e}}_{i}, A$ and $C$ are the bend and twist elastic constants, respectively, and $\Lambda(s)$ is a Lagrange multiplier function enforcing local inextensibility.

A "natural" orthonormal frame [13] of a given curve consists of the unit tangent and two other vectors constrained to have zero tangential rotation rate. Imagine a weather vane sliding along the curve and always lying in the normal plane [16]. If the weather vane spins around $\hat{\mathbf{e}}_{3}$ with vanishing initial tangential angular momentum then it traces out one member of a family of natural frames related by $s$-independent rotations. Such a frame is constructed simply by untwisting the material frame, i.e., rotating $\hat{\mathbf{e}}_{1}$ and $\hat{\mathbf{e}}_{2}$ around $\hat{\mathbf{e}}_{3}$ by minus the accumulated twist angle $\vartheta(s) \equiv \int^{s} d s^{\prime} \Omega_{3}$. Since $\boldsymbol{\epsilon}^{*} \cdot \partial_{s} \boldsymbol{\epsilon}=0$, the vector $\boldsymbol{\epsilon} \equiv\left(\hat{\mathbf{e}}_{1}+i \hat{\mathbf{e}}_{2}\right) \exp i \vartheta$ does not twist around $\hat{\mathbf{e}}_{3}$.

The rotation rate of this frame is given by the complex curvature $\psi: \partial_{s} \boldsymbol{\epsilon}=-\psi \hat{\mathbf{e}}_{3}$ and $\partial_{s} \hat{\mathbf{e}}_{3}=\operatorname{Re}\left(\psi \boldsymbol{\epsilon}^{*}\right)$, where

$$
\psi=\left(-i \Omega_{1}+\Omega_{2}\right) \exp i \int^{s} d s^{\prime} \Omega_{3} .
$$

Some elementary shapes have simple $\psi$ representations: the straight line has $\psi=0$, and the helix $\psi=a e^{i p s}(a, p$ are real constants). Likewise, certain geometric properties of curves are easily stated in terms of $\psi$ : a curve lies in a plane if and only if $(\operatorname{Re} \psi(s), \operatorname{Im} \psi(s))$ lies on a line through the origin in the complex $\psi$ plane, and a curve lies on a sphere if and only if $(\operatorname{Re} \psi(s), \operatorname{Im} \psi(s))$ lies on a line not through the origin [17].

We compute from (1) the elastic forces and moments per unit length using the principle of virtual work. If $\mathbf{F}$ is the force acting on a cross section, the force per unit length $\mathbf{F}_{s}$ is found by varying the position of the centerline without rotating any element of the rod about the tangent direction: $\delta \mathcal{E}=-\int d s \mathbf{F}_{s} \cdot \delta \mathbf{r}(s)$, with the rotation angle $\delta \chi \equiv \hat{\mathbf{e}}_{2} \cdot \delta \hat{\mathbf{e}}_{1}=0$. Likewise, if $\mathbf{M}$ is the moment acting on a cross section, the tangential component of the moment per unit length $\mathbf{M}_{s}$ is found by varying the orientations of the elements of the rods without moving the centerline: $\delta \mathcal{E}=-\int d s \mathbf{M}_{s} \cdot \hat{\mathbf{e}}_{3} \delta \chi$, with $\delta \mathbf{r}=0$. Using $\delta \mathbf{r}_{s} \equiv(\delta \mathbf{r})_{s}, \delta \hat{\mathbf{e}}_{a}=(\delta \chi) \boldsymbol{\epsilon}_{a b} \hat{\mathbf{e}}_{b}-\left(\hat{\mathbf{e}}_{a} \cdot \delta \mathbf{r}_{s}\right) \hat{\mathbf{e}}_{3}$ for $a, b=1,2$ and $\delta \hat{\mathbf{e}}_{3}=\delta \mathbf{r}_{s}-\left(\hat{\mathbf{e}}_{3} \cdot \delta \mathbf{r}_{s}\right) \hat{\mathbf{e}}_{3}$, we find

$$
\begin{gathered}
\delta \psi=-2 \psi \hat{\mathbf{e}}_{3} \cdot \delta \mathbf{r}_{s}+\epsilon \cdot \delta \mathbf{r}_{s s} \\
-i \psi \operatorname{Im} \int^{s} d s^{\prime} \psi \epsilon^{*} \cdot \delta \mathbf{r}_{s}, \\
\delta \Omega_{3}=\delta \chi_{s}-\Omega_{3} \hat{\mathbf{e}}_{3} \cdot \delta \mathbf{r}_{s}+\left(\Omega_{1} \hat{\mathbf{e}}_{1}+\Omega_{2} \hat{\mathbf{e}}_{2}\right) \cdot \delta \mathbf{r}_{s} .
\end{gathered}
$$

Note that keeping $\delta \chi=0$ during the variation does not imply that the twist rate $\Omega \equiv \Omega_{3}$ stays fixed! (For brevity we drop the subscript on $\Omega_{3}$ below.)

Thus the force per unit length is $-\delta \mathcal{E} / \delta \mathbf{r}=$ $-\mathbf{e}_{3} \Lambda_{s}+\operatorname{Re}\left(\boldsymbol{\epsilon}^{*} \mathcal{F}_{\perp}\right)$, where

$$
\mathcal{F}_{\perp}=-A\left(\psi_{s s}+\frac{1}{2}|\psi|^{2} \psi\right)+i C(\Omega \psi)_{s}-\Lambda \psi,
$$

and a term $C \Omega^{2} / 2$ has been absorbed into $\Lambda$. Separating (5) into its real and imaginary parts yields the classic results of Love [18]. Note that the force density is invariant under $\psi \mapsto e^{i \phi} \psi, \boldsymbol{\epsilon} \mapsto e^{i \phi} \boldsymbol{\epsilon}$ for constant $\phi$. The tangential moment per unit length is $-\delta \mathcal{E} / \delta \chi(s)=$ $C \Omega_{s}$. The conditions of zero force and moment at free ends imply the boundary conditions $\psi=\psi_{s}=\Lambda=$ $\Omega=0$.

The dynamics of $\psi$ and $\Omega$ follow immediately from (4) by choosing $\delta \mathbf{r}=\mathbf{r}_{t} \delta t$ and $\delta \chi=\chi_{t} \delta t$. Their form is completely dictated by geometry. (That for $\psi_{t}$ is equivalent to earlier results [15]; the undamped analog of the $\Omega_{t}$ equation appears in [8].) The right-hand side of (4) expresses a fact easily demonstrated with the nearest rubber tube; there are two distinct ways of changing the twist density: (i) nonuniform axial rotations of the rod and (ii) nonplanar bending motions.

To complete the equations of motion, we use the simplest Rouse dynamics in which the only effect of the surrounding fluid is to provide a local isotropic drag [19] proportional to the local velocity: $\mathbf{f}_{\mathrm{drag}}=\zeta \mathbf{r}_{t}$. Likewise, we take the tangential moment per unit length to be $m_{\mathrm{drag}}=\zeta_{r} \chi_{t}$. Since at zero Reynolds number the elastic forces and moments must exactly balance the drag forces and moments, the complex curvature and twist obey

$$
\begin{gathered}
\zeta \psi_{t}=\left(\partial_{s}^{2}+|\psi|^{2}\right) \mathcal{F}_{\perp}+i \psi \operatorname{Im} \int^{s} d s^{\prime} \mathcal{F}_{\perp}{ }^{*} \psi_{s} \\
-\Lambda_{s} \psi_{s}, \\
\zeta_{r} \Omega_{t}=C \Omega_{s s}+\left(\zeta_{r} / \zeta\right) \operatorname{Im} \mathcal{F}_{\perp s}^{*} \psi .
\end{gathered}
$$

Local inextensibility, $\partial_{t} d s=0, \quad$ requires $\Lambda_{s s}=$ $-\operatorname{Re}\left(\psi^{*} \mathcal{F}_{\perp}\right)$. Equation (6) is covariant and (7) invariant under $s$-independent rotations of the natural basis about the tangent $\psi \mapsto e^{i \phi} \psi$. Since the elastic energy (1) is achiral, the dynamics are covariant under mirror reflections: $\psi \mapsto \psi^{*}, \Omega \mapsto-\Omega$. Equations (6) and (7) describe the filament's changes in shape and twist without relation to an external coordinate system, and so are intrinsic; rigid translations and rotations do not enter the dynamics since $\mathbf{r}_{t}$ and $\chi_{t}$ appear only through their $s$ derivatives.

The derivation of the above dynamics relies solely on geometry and mechanics. However, for closed curves, we can reconsider (7) in light of the celebrated relation $\mathcal{L} k=\mathcal{T} w+\mathcal{W} r$ [20]. Here $\mathcal{L} k$ is the linking number of the two curves traced out by the heads of the vectors 
$\hat{\mathbf{e}}_{1}$ and $\hat{\mathbf{e}}_{2}$ (assuming a continuous material frame), $\mathcal{T} w \equiv$ $\int d s \Omega / 2 \pi$, and the writhe $\mathcal{W} r$ is an integer plus $A / 2 \pi$, where $A$ is the signed area enclosed by the curve swept out on the unit sphere by $\hat{\mathbf{e}}_{3}$ [21]. For a locally inextensible filament, the twist dynamics (7) can be interpreted as a local link conservation law under the assumption that smooth filament motions will not change the integer contribution to the writhe [9]. The time derivative of the density $w r$ of the noninteger contribution to the writhe is $\partial_{t} w r=\hat{\mathbf{e}}_{3} \cdot \partial_{t} \hat{\mathbf{e}}_{3} \times \partial_{s} \hat{\mathbf{e}}_{3} / 2 \pi=-\operatorname{Im}\left(\mathcal{F}_{\perp s}{ }^{*} \psi\right) / 2 \pi \zeta$. Therefore, the twist dynamics has the form $\rho_{t}+j_{s}=$ 0 , with the "link density" $\rho=\Omega / 2 \pi+w r$ and link current $j=-\left(C / \zeta_{r}\right) \Omega_{s}$. Of course, since the link is not a single integral of a local density there is no true link density; however, changes in link can be written as a single integral over a density [21]. While $\mathcal{L} k$ and $\mathcal{W r}$ are conventionally defined only for closed filaments, the equations of motion (6) and (7) show that the tradeoff between twist and writhe still has meaning for open filaments.

With the coupled evolution equations for $\psi$ and $\Omega$ we turn to linear and nonlinear aspects of supercoiling instabilities. The simplicity of the $\psi$ formulation for open filaments is seen first in the stability of a straight rod with a uniform twist density (temporarily neglecting the boundary conditions on $\Omega$ ). The linearization of (6) is

$$
\zeta \psi_{t}=-A \psi_{s s s s}+i C \Omega \psi_{s s s} .
$$

The eigenfunctions $W_{n}$ of $-A \partial_{s}^{4}+i C \Omega \partial_{s}^{3}$ have eigenvalues $\sigma_{n}=-A k_{n}^{4}+C \Omega k_{n}^{3}$ (and associated growth rates $\sigma_{n} / \zeta$ ) which are real since the operator is Hermitian. If $\Omega=0$, the modes are planar shapes with $W_{n}=c_{n}^{(1)} \sin \left(k_{n} s\right)+c_{n}^{(2)} \cos \left(k_{n} s\right)+c_{n}^{(3)} \sinh \left(k_{n} s\right)+$ $c_{n}^{(4)} \cosh \left(k_{n} s\right)$, with $\cos \left(k_{n} L\right) \cosh \left(k_{n} L\right)=1$ [12]. Since the $W_{n}(s)$ satisfy the boundary conditions and form a complete set, the shape of any free elastica in three dimensions, whether tightly wound helices or plectonemes, is expressible as

$$
\psi(s, t)=\sum_{n=1}^{\infty} C_{n}(t) W_{n}(s) .
$$

The time evolution is a nonlinear dynamical system in $C_{n}(t) \equiv\left\langle W_{n} \mid \psi(t)\right\rangle$, where the $W_{n}$ are normalized.

The linear stability analysis of a free rod with uniform twist is identical to that of a clamped rod with uniform twist. Using the complex Monge representation $\xi(z)=$ $x(z)+i y(z)$, where $\mathbf{r}(z)=[x(z), y(z), z]$, yields $\zeta \xi_{t}=$ $-A \xi_{z z z z}+i C \Omega \xi_{z z z}$ with $\xi_{z}=\xi_{z z}=0$ at the clamped ends. Note, however, that the Monge representation is incapable of describing plectonemes. The critical condition for the first centerline instability is $\Omega_{c} \approx 8.98 \mathrm{~A} / C L$ [12]. When the twist is nonzero, each eigenfunction is a superposition of four helices with appropriate spatial damping: $W_{n}(s)=\sum_{\mu=1}^{4} c_{n}^{(\mu)} \exp \left(i k_{n}^{(\mu)} s\right)$, where for each $n$ the four $k_{n}^{(\mu)}$ are in general complex. Modes with negative eigenvalue $\sigma_{n}$ have three helices of one handedness and
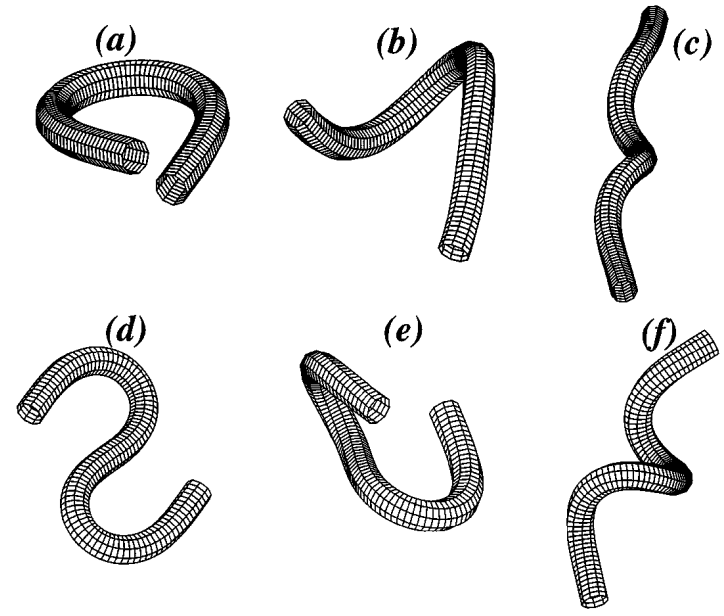

FIG. 2. Filament shapes at various twist densities. (a)-(c): $\psi=10 W_{1}$ at $\Omega / \Omega_{c}^{(1)}=0.22,1.0,2.22 ;(\mathrm{d})-(\mathrm{f}): \psi=8 W_{2}$ at $\Omega / \Omega_{c}^{(2)}=0.13,0.58,1.29$.

one of the other; otherwise, all have the same handedness. Shapes calculated from the first two modes are shown in Fig. 2.

The writhe-tracking term in (7) is explicitly nonlinear; when the diffusion constant $C / \zeta_{r}=0$, twist changes only when the filament writhes. In assessing the relative importance of twist diffusion and writhe tracking we note that while $\zeta_{r}$ in (7) was interpreted as a rotational drag coefficient, in the context of bacterial filaments it is not clear that this dissipative mechanism dominates others, e.g., rearrangement of the polymer network inside the cell walls. If the shape instabilities are generated by nonequilibrium twist, then twist must not relax on time scales shorter than the observed buckling time (tens of minutes [22]). Using the purely hydrodynamic twist diffusion constant $C / \zeta_{r}$ $[10,23]$ (with $C \equiv 10^{-10} \mathrm{erg} \mathrm{cm} \mathrm{[24]} \mathrm{and} \zeta_{r} \equiv 10^{-10} \mathrm{erg}$ $\mathrm{sec} / \mathrm{cm}$ for a $1 \mu \mathrm{m}$ diameter slender rod in water), and the typical filament length $L \sim 100 \mu \mathrm{m}$ for buckling, the time scale $\tau_{D}=L^{2} \zeta / C$ for twist to diffuse out the ends of the filament is only $10^{-4} \mathrm{sec}$, eliminating hydrodynamic drag as a candidate mechanism. This and other observations (e.g., of slow stress relaxation [24]) strongly suggest a dominant dissipative mechanism within the filament, characterized by a very much larger $\zeta_{r}$. Therefore,

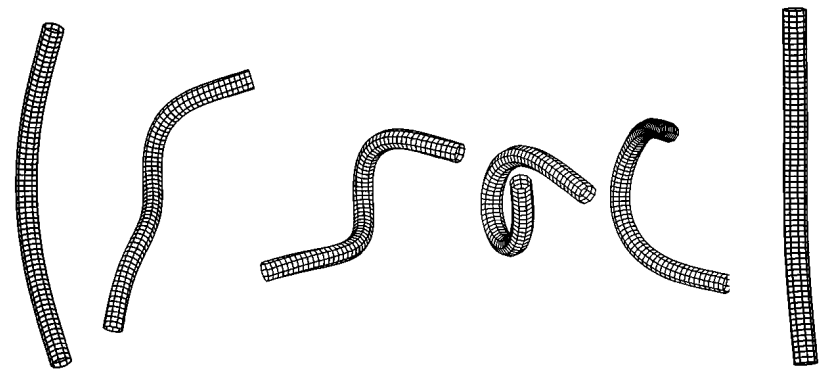

FIG. 3. Geometric untwisting. Filament shapes are calculated from numerical integration of (6) and (7) with $C / A=1$. Time proceeds from left to right. 

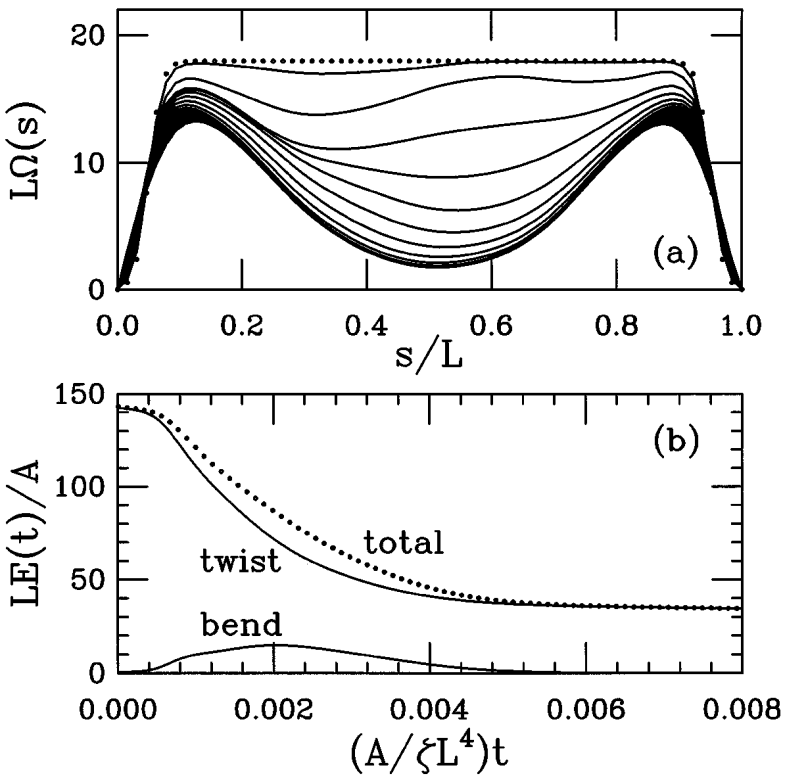

FIG. 4. Details of geometric untwisting. (a) Twist density evolution, with dotted line showing initial profile. Asymmetry in $\Omega$ arises from that of the initial shape. (b) Time evolution of twist, bend, and total elastic energy.

we consider the limit of negligible diffusion constant $C / \zeta_{r}$, where twist changes only by bending rather than by axial rotation, a relaxation mechanism we term "geometric untwisting."

Geometric untwisting is illustrated in Fig. 3, obtained by numerical integration [25] of (6) and (7). An additional nonlocal contact force in $\mathcal{F}_{\perp}$ prevents self-crossing. The initial condition is a nearly straight filament with twist density of the form shown in Fig. 4(a). This mesalike profile reflects uniform local twist production during growth and diffusive boundary layers at the ends [26]. Motion begins as chiral buckling; as the curvature increases, twist is converted to writhe, and $\Omega$ decreases in the interior [Fig. 4(a)]. When twist has sufficiently relaxed and writhe is of order unity, the filament relaxes back to the straight ground state as link flows out the ends. In this overdamped dynamics the total elastic energy is monotonically decreasing, despite the transient increase in the bending energy during loop formation [Fig. 4(b)].

The sequence in Fig. 3 is quite similar to that exhibited by short filaments of $B$. subtilis [22]: after beginning the transition to a plectoneme via a looping instability, the ends touch only transiently, the loop snaps open, and the filament straightens. Plectoneme formation occurs after several aborted looping events, presumably as the twist stresses continually increase with filament extension.

The results presented here proceed from the robust to the specific: from kinematics dictated solely by geometry, through viscous dynamics governed by elasticity, and united in a description of the onset of bacterial supercoiling. This particular union will help unravel the puzzles surrounding growth-induced iterated writhing instabili- ties of bacterial fibers [26] and elucidate possible twistinduced instabilities in related systems, such as thermally fluctuating nucleic acids [27].

We are indebted to Neil Mendelson and Charles Wolgemuth for ongoing collaborations, and thank F. Gittes, A. Goriely, G. Huber, R. Kamien, J. Kessler, P. Nelson, and M. Tabor. This work was supported by the NSF (R. E. G.).

[1] D'Arcy W. Thompson, On Growth and Form (The University Press, Cambridge, 1942).

[2] D. Riveline, C.H. Wiggins, R. E. Goldstein, and A. Ott, Phys. Rev. E 56, 1330 (1997); C. H. Wiggins, D. Riveline, R. E. Goldstein, and A. Ott, Biophys. J. 74, 1043 (1998).

[3] N. H. Mendelson, Proc. Natl. Acad. Sci. U.S.A. 73, 1740 (1976); Microbiol. Rev. 46, 341 (1982).

[4] M. J. Tilby, Nature (London) 266, 450 (1977).

[5] B. Alberts, D. Bray, J. Lewis, M. Raff, K. Roberts, and J.D. Watson, Molecular Biology of the Cell (Garland Publishing, Inc., New York, 1994), 3rd ed.

[6] I. Klapper, J. Comput. Phys. 125, 325 (1996); A. Goriely and M. Tabor, Phys. Rev. Lett. 77, 3537 (1996).

[7] J.F. Marko and E.D. Siggia, Macromolecules 27, 981 (1994).

[8] I. Klapper and M. Tabor, J. Phys. A 27, 4919 (1994).

[9] R. D. Kamien, Eur. Phys. J. B 1, 1 (1998).

[10] A. C. Maggs, cond-mat/9704054.

[11] G. Kirchhoff, J. F. Math (Crelle) 50, 285 (1859).

[12] See, e.g., L.D. Landau and E. M. Lifshitz, Theory of Elasticity (Pergamon Press, Oxford, 1986), 3rd ed.

[13] G. Darboux, Leçons sur la Théorie Genérale des Surfaces (Gauthier-Villars, Paris, 1915), Vol. I, p. 22.

[14] H. Hasimoto, J. Fluid Mech. 51, 477 (1972).

[15] R. E. Goldstein and S. A. Langer, Phys. Rev. Lett. 75, 1094 (1995); K. Nakayama, H. Segur, and M. Wadati, Phys. Rev. Lett. 69, 2603 (1992).

[16] Joel Langer (private communication).

[17] R. L. Bishop, Am. Math. Monthly 82, 246 (1975).

[18] A.E.H. Love, A Treatise on the Mathematical Theory of Elasticity (Cambridge, London, 1965), 4th ed. See also Y. Shi and J. E. Hearst, J. Chem. Phys. 101, 5186 (1994).

[19] M. Doi and S.F. Edwards, The Theory of Polymer Dynamics (Oxford University Press, New York, 1986).

[20] G. Căluguăreanu, Rev. Math. Pures Appl. 4, 5 (1959); J. H. White, Am. J. Math. 91, 693 (1969); F. B. Fuller, Proc. Nat. Acad. Sci. U.S.A. 68, 815 (1971).

[21] F. B. Fuller, Proc. Natl. Acad. Sci. U.S.A. 75, 3557 (1978).

[22] N. H. Mendelson, J. J. Thwaites, J. O. Kessler, and C. Li, J. Bacteriol. 177, 7060 (1995).

[23] M. D. Barkley and B. H. Zimm, J. Chem. Phys. 70, 2991 (1979); J. M. Schurr, Chem. Phys. 84, 71 (1984).

[24] J. J. Thwaites and N.H. Mendelson, Int. J. Biol. Macromol. 11, 201 (1989).

[25] Using methods similar to T. Y. Hou, J. S. Lowengrub, and M. J. Shelley, J. Comp. Phys. 114, 312 (1994).

[26] C. H. Wiggins et al. (to be published).

[27] L. F. Liu and J. C. Wang, Proc. Natl. Acad. Sci. U.S.A. 84, 7024 (1987); C. Levinthal and H. R. Crane, ibid. 42, 436 (1956). 